\title{
Vector analyzing power in elastic electron-proton scattering
}

\author{
L. Diaconescu ${ }^{1}$ and M. J. Ramsey-Musolf ${ }^{1,2}$ \\ ${ }^{1}$ Kellogg Radiation Laboratory, California Institute of Technology, Pasadena, California 91125, USA \\ ${ }^{2}$ Department of Physics, University of Connecticut, Storrs, Connecticut 06269, USA
}

(Received 18 May 2004; published 11 November 2004)

\begin{abstract}
We compute the vector analyzing power (VAP) for the elastic scattering of transversely polarized electrons from protons at low energies using an effective theory of electrons, protons, and photons. We study all contributions through second order in $E / M$, where $E$ and $M$ are the electron energy and nucleon mass, respectively. The leading-order VAP arises from the imaginary part of the interference of one- and two-photon exchange amplitudes. Subleading contributions are generated by the nucleon magnetic moment and charge radius as well as recoil corrections to the leading-order amplitude. Working to $O(E / M)^{2}$, we obtain a prediction for $A_{n}$ that is free of unknown parameters and that agrees with the recent measurement of the VAP in backward angle ep scattering.
\end{abstract}

DOI: 10.1103/PhysRevC.70.054003

PACS number(s): 11.30.Er, 14.20.Dh, 25.30.Bf

\section{INTRODUCTION}

The study of the vector analyzing power (VAP), $A_{n}$, in polarized electron-proton scattering has recently become a topic of considerable interest in nuclear physics. The VAP is a time-reversal $(\mathrm{T})$ odd, parity $(\mathrm{P})$ even correlation between the electron spin and the independent momenta associated with the scattering process,

$$
A_{n} \sim \epsilon^{\mu \nu \alpha \beta} P_{\mu} S_{\nu} K_{\alpha} K_{\beta}^{\prime},
$$

where $S, P$, and $K\left(K^{\prime}\right)$ denote the electron spin, initial proton momentum, and incident (scattered) electron momentum, respectively. A nonzero VAP cannot arise at leading-order in quantum electrodynamics (QED), but could be generated by new T-odd, P-even interactions involving electrons and quarks. Searches for such interactions have been carried out in neutron and nuclear $\beta$-decay as well as nuclear $\gamma$-decays [1-3]. Indirect constraints may also be obtained from limits on the permanent electric dipole moments of neutral atoms under various assumptions regarding the pattern of symmetry-breaking [4-8]. The sensitivity of direct searches for T-odd, P-even interactions is generally limited by the presence of QED "final state interactions" (FSIs) that break the T-symmetry between initial and final states and give rise to nonvanishing T-odd, P-even observables. Uncertainties in theoretical calculations of these final state interactions would cloud the interpretation of a sufficiently precise T-odd, P-even measurement in terms of new interactions. Observations of T-odd, P-even correlations in nuclear $\gamma$-decays are consistent with theoretical calculations of QED final state interactions [9], while T-odd, P-even searches in neutron $\beta$-decay have yet to reach the sensitivity needed to discern these effects.

Recently, the SAMPLE Collaboration has reported a nonzero measurement of the VAP in polarized, elastic electronproton scattering [10], making it the first nonzero result for any T-odd, P-even observable in any electron scattering process. The result has received widespread attention, as it differs substantially from the simplest theoretical estimate of QED final state contributions that neglects proton recoil and internal structure [11]. While one might speculate that this difference reflects the presence of new physics, a more likely explanation lies in elements of nucleon structure omitted from the simplest treatments of QED FSIs.

If so, then the SAMPLE result, as well as other VAP measurements that have been completed or are under consideration, could have important implications for the interpretation of other precision observables involving hadrons that require computation of QED corrections to the leading-order amplitude. Such observables include the ratio of proton electromagnetic form factors obtained via Rosenbluth separation in elastic ep scattering [12], higher-order "box graph" contributions to weak interaction observables [13], or QED final state interactions in direct searches for T-odd, P-even effects. In each instance, a calculation of QED corrections requires a realistic and sufficiently precise treatment of hadronic intermediate states, particularly those arising in two-photon exchange amplitudes, $\mathcal{M}_{\gamma \gamma}$, or the analogous amplitudes involving the exchange of one heavy gauge boson and one photon. Since the leading QED contribution to $A_{n}$ arises from $\operatorname{Im} \mathcal{M}_{\gamma \gamma}$, experimental measurements of the VAP provide an important test of theoretical calculations of $\mathcal{M}_{\gamma \gamma}$ needed for the interpretation of other measurements.

At the same time, the VAP provides a new window on nucleon structure, as $\mathcal{M}_{\gamma \gamma}$ probes the doubly virtual Compton scattering (VVCS) amplitude. In recent years, virtual Compton scattering (VCS) on the proton has become an important tool in probing the internal structure of the proton. VCS involves the coupling of one virtual and one real photon to a hadronic system. In the case of the proton, the VCS cross section is sensitive to the generalized polarizabilities of the proton, and its measurement should provide insight into the proton structure [14]. In practice, however, this cross section includes Bethe-Heitler (BH) amplitudes associated with radiation of a real photon from the electrons. Proper treatment of the cross section must therefore be taken in order to obtain a correct interpretation of the measurement. In contrast, the process involving the coupling of two virtual photons to the hadronic system is immune to background $\mathrm{BH}$ amplitudes and, thus, offers an alternative to VCS in probing the proton structure. 


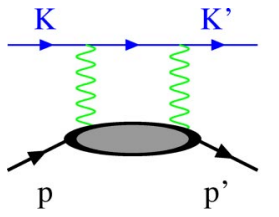

(a)

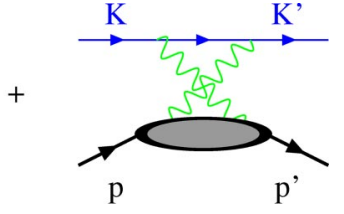

(b)
FIG. 1. (Color online) Two photon exchange diagrams. The wavy lines indicate virtual photons, while $k\left(k^{\prime}\right)$ and $p\left(p^{\prime}\right)$ denote the initial (final) electron and proton momenta, respectively.

With the aforementioned motivation in mind, we study the VAP in the framework of an effective theory of lowenergy ep scattering. Since the SAMPLE measurement corresponds to kinematics close to the pion electroproduction threshold, we consider only the electron, photon, and nucleon as dynamical degrees of freedom. In this respect, our analysis corresponds to the use of heavy baryon chiral perturbation theory with the pions integrated out. To make the treatment systematic, we expand $A_{n}$ in powers of $p / M$, where $p$ is either the incident electron energy $(E)$ or mass $(m)$ and $M$ is the nucleon mass. Working to second order in $p / M$, we obtain all contributions to $A_{n}$ that arise uniquely from one-loop, two-photon exchange amplitudes and obtain a prediction that is free from any unknown parameters. We also write down the leading, nonrenormalizable T-odd, P-even eepp operators whose intereference with $\mathcal{M}_{\gamma}$ can generate a nonzero VAP and show that they contribute at $O(p / M)^{4}$.

We find that inclusion of all one-loop effects through $O(p / M)^{2}$ in $\mathcal{M}_{\gamma \gamma}$ as well as all terms in $\mathcal{M}_{\gamma}$ through this order is sufficient to resolve the disagreement between the SAMPLE result and the simplest potential scattering predictions. This resolution follows from several effects that occur beyond leading order in $p / M$ : recoil corrections to the pure charge scattering result obtained in Ref. [11], the nucleon isovector magnetic moment, and the proton charge radius. In the absence of dynamical pions, contributions from the nucleon polarizability arise at higher order than we consider here and appear unnecessary to account for the experimental result. Given that the incident electron energy $E$ is of the same order as $m_{\pi}$, we have no a priori reason to expect agreement of our computation with experiment. What it suggests, however, is that for this kinematic regime, pions play a less important role in the VVCS amplitude than one might naively expect. Future low-energy $A_{n}$ measurements, taken over a broader range in $q^{2}$ and scattering angle than relevant to the SAMPLE measurement, would provide additional, useful tests of this conclusion.

We also consider $A_{n}$ at forward scattering angles and energies somewhat higher than those of the SAMPLE experiment, since preliminary results for this kinematic domain have been reported by the A4 Collaboration at the MAMI facility in Mainz [15]. Although we would not expect our framework to be reliable in this kinematic regime, where the electron energy $E$ is much closer to $M$, it is nonetheless instructive to compare with the Mainz preliminary results as a way of pointing to the physics that may be operative in this domain. Indeed, we find substantial disagreement with the

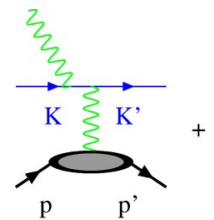

(a)

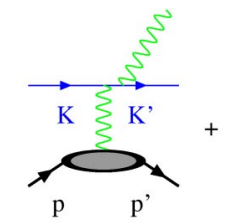

(b)

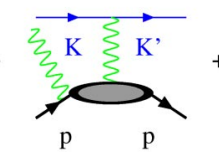

(c)

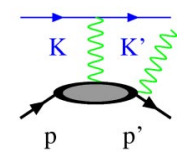

(d)
FIG. 2. (Color online) Bremsstrahlung contributions. Labels are the same as in Fig. 1.

preliminary Mainz data. The culprit could be that going to the Mainz kinematics exceeds the limit of validity of our effective theory, that we must include additional dynamical degrees of freedom such as the $\pi$ or $\Delta(1230)$ resonance, or both. Future studies using alternative methods such as dispersion relations may be needed to explore this kinematic domain.

Finally, we also consider $A_{n}$ for polarized Møller scattering. The VAP for this process has been measured by the E158 Collaboration at SLAC [16], and theoretical computations given in Refs. [17-19]. Our computation agrees with these earlier $A_{n}(e e)$ calculations, providing a useful crosscheck on our study of the VAP for ep scattering.

Our discussion of these points is organized in the remainder of the paper as follows. In Sec. II, we discuss general features of $A_{n}$ and our approach to the computation. Section III provides details of the calculation. In Sec. IV, we give numerical results and discuss their significance, while Sec. V gives our conclusions. Technical details are provided in the Appendixes.

\section{GENERAL CONSIDERATIONS}

We are interested in computing the VAP in elastic ep scattering:

$$
A_{n}=\frac{d \sigma_{\uparrow}-d \sigma_{\downarrow}}{d \sigma_{\uparrow}+d \sigma_{\downarrow}}=\frac{2 \operatorname{Im} \mathcal{M}_{\gamma \gamma}^{*} \mathcal{M}_{\gamma}}{\left|\mathcal{M}_{\gamma}\right|^{2}}
$$

where $d \sigma_{\uparrow(\downarrow)}$ is the differential cross section for scattering of electrons with incident spin parallel (antiparallel) to $\vec{K} \times \vec{K}^{\prime}$. In a phase convention where the single $\gamma$-exchange amplitude $\mathcal{M}_{\gamma}$ is purely real, $A_{n}$ requires a nonvanishing imaginary part of $\mathcal{M}_{\gamma \gamma^{\circ}}{ }^{1}$ To compute the latter, one must consider both the box and crossed-box diagrams of Fig. 1. Simple power-counting arguments indicate that the contribution to $\mathcal{M}_{\gamma \gamma}$ arising from the leading-order $\gamma p$ couplings is ultraviolet finite but infrared divergent. Thus, in general, one must also compute the contributions to $A_{n}$ arising from the bremsstrahlung diagrams of Fig. 2. As we show by explicit calculation in Appendix A, however, the bremsstrahlung contribution to $A_{n}$ vanishes identically, while $\operatorname{Im} \mathcal{M}_{\gamma \gamma}$ is infrared finite. The resulting, leading-order contribution to $A_{n}$ is $O(p / M)^{0}$.

\footnotetext{
${ }^{1}$ By $\operatorname{Im} \mathcal{M}_{\gamma \gamma}$, we mean the coefficients of the various products of fermion bilinears, $\bar{e} \Gamma e \bar{N} \Gamma^{\prime} N$, etc. that appear in the amplitude.
} 
Additional contributions to $\mathcal{M}_{\gamma \gamma}$ arise from higher-order operators that couple one or more virtual photons to the proton and electron. We neglect the latter since they are suppressed by additional powers of the fine structure constant. ${ }^{2}$ In contrast, the $\gamma p$ operators are induced by strong interactions and have couplings of order $e$. In order to treat their contributions systematically, we adopt an effective theory framework since we cannot compute the operator coefficients from first principles in quantum chromodynamics. The natural framework for doing so is heavy baryon chiral perturbation theory $(\mathrm{HB} \chi \mathrm{PT})$, which provides a systematic expansion in powers of $p / \Lambda_{\chi}$ and $p / M$, where $\Lambda_{\chi}=4 \pi F_{\pi}$ is the scale of chiral symmetry-breaking and $p$ is an external momentum or mass with magnitude much less than $M$ and $\Lambda_{\chi}$. In the present case, where we integrate out the pions, we take $p=E$ or $m$ and use $M$ as the heavy scale. For the kinematics of the SAMPLE experiment, $E>>m$. Since there are no hard collinear infrared singularities in $\operatorname{Im} \mathcal{M}_{\gamma \gamma}$, we may drop all power corrections involving the electron mass and obtain our result as an expansion in $E / M$.

The leading terms in a heavy baryon Lagrangian for nucleons and photons relevant to our computation are

$$
\begin{aligned}
\mathcal{L}_{N \gamma}= & \bar{B}_{v} i v \cdot D B_{v}+\frac{1}{2 M} \bar{B}_{v}\left[(v \cdot D)^{2}-D^{2}\right] B_{v} \\
& +\frac{e \mu}{2 M} \epsilon_{\mu \nu \alpha \beta} F^{\mu \nu} v^{\alpha} \bar{B}_{v} S^{\beta} B_{v}-\frac{e C_{r}}{M^{2}} \bar{B}_{v} v_{\mu} B_{v} \partial_{\lambda} F^{\mu \lambda}+\cdots
\end{aligned}
$$

where $B_{v}$ is the field for a heavy proton of velocity $v_{\mu}$, where $D_{\mu}=\partial_{\mu}-i e A_{\mu}$, and where we have shown explicitly all $\gamma p$ interactions through $O\left(p^{3}\right)$. The latter arise from the subleading kinetic term in Eq. (3) as well as from the operators containing the field strength, $F^{\mu \nu}$. The coefficient $\mu=2.793$ is the proton magnetic moment, while $C_{r}$ determines the proton Sachs, or electric, radius,

$$
C_{r}=\frac{M^{2}}{6}\left\langle r^{2}\right\rangle_{E}=\left.M^{2} \frac{d G_{E}^{p}(t)}{d t}\right|_{t=0},
$$

where $t=q^{2}$. The experimental value for $\left\langle r^{2}\right\rangle_{E}=0.743 \mathrm{fm}^{2}$ $[20,21]$ implies $C_{r}=2.81$. When included in the loop diagrams of Fig. 1, these interactions generate contributions to the ep amplitude $M_{\gamma}$ and $M_{\gamma \gamma}$ through order $(p / M)^{2}$ relative to the leading term. To this order, operators associated with the nucleon polarizability [see Fig. 3(e)] do not contribute, as they occur at $O\left(p^{4}\right)$ in $\mathcal{L}_{N \gamma}$ when the pion is treated as heavy.

Higher-order contributions to $A_{n}$ can also arise from effective T-odd, P-even $e e N N$ interactions. The origin of such operators could be either physics that we have integrated out, such as contributions to $\mathcal{M}_{\gamma \gamma}$ from $\pi N$ or $\Delta$ intermediate states, or explicit T-odd, P-even interactions arising from new physics. As shown in Appendix B, there exist no Hermitian, four-fermion operators at dimension 6 that contribute to $A_{n}$. The lowest dimension T-odd, P-even four-fermion op-

\footnotetext{
${ }^{2}$ For high energy scattering, these higher-order QED contributions may receive logarithmic enhancements [19].
}

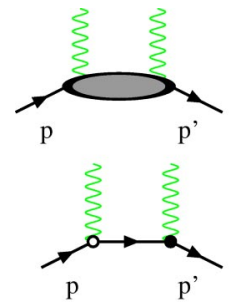

(c)

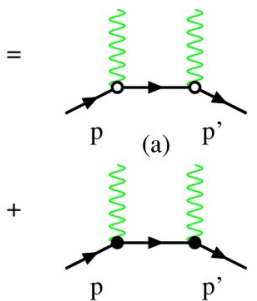

(d)

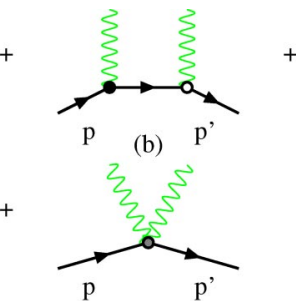

(e)
FIG. 3. (Color online) Contributions to the VVCS amplitude appearing in Fig. 1. Open circles indicate the leading-order $\gamma N$ couplings, while dark circles indicate higher-order couplings, such as the magnetic moment and charge radius. Shaded circle denotes that nucleon polarizability operator.

erators have dimension 7 and would nominally contribute to $A_{n}$ at $O(p / M)^{3}$. We show, however, that contributions from these operators vanish to this order and first arise at $O(p / M)^{4}$. Since we truncate our analysis at two orders lower, we may neglect these operators and obtain a parameter-free prediction for the VAP. Nevertheless, we discuss these operators briefly in Sec. IV when considering the possible size of neglected, higher-order contributions. ${ }^{3}$

As we show in detail in Sec. III, the leading one-loop contributions to $A_{n}$-generated by two $O(p) \gamma p$ insertions in the VVCS amplitude [Fig. 3(a)] —are finite, non-analytic in $p$, and occur $O(p / M)^{0}$, whereas those generated by the dimension-7 T-odd, P-even operators arise at $O(p / M)^{4}$. Thus, the leading contributions are uniquely determined from the one-loop calculation. Similarly, contributions to $\mathcal{M}_{\gamma \gamma}$ involving one $O(p)$ and one $O\left(p^{2}\right) \gamma p$ interaction [Figs. 3(b) and 3(c)] contribute to $A_{n}$ at $O(p / M)$, are also finite and nonanalytic in $p$, and are unique to the loop calculation. The $O(p / M)^{2}$ loop contributions arise either from two $O\left(p^{2}\right) \gamma p$ operators [e.g., two insertions of the nucleon magnetic moment operator, Fig. 3(d)] or one $O(p)$ and one $O\left(p^{3}\right)$ term (viz., the proton charge radius). We find, however, that the $O(p / M)^{2}$ components of $\mathcal{M}_{\gamma \gamma}$ arise only from the $\gamma p$ magnetic moment interaction as well as from recoil order terms in $\mathcal{L}_{N \gamma}$. Contributions to $\mathcal{M}_{\gamma \gamma}$ from the proton charge radius vanish, though it does contribute to $A_{n}$ as a higher-order term in $\mathcal{M}_{\gamma}$.

\section{TWO-PHOTON EXCHANGE}

The evaluation of four-point functions for general kinematics does not readily lend itself to evaluation using standard Feynman parametrization in the loop integrals. Alternate methods for evaluating these integrals that do not rely explicitly on Feynman parameters have been worked out in Refs. [23,24] and have become standard. In the present case, where we are interested in backward angle scattering at nonzero $q^{2}$, we would ideally like to use this formalism. However, the form of the heavy baryon propagator does not per-

\footnotetext{
${ }^{3}$ For an earlier, phenomenological calculation that includes some of these higher order contributions, see Ref. [22].
} 
mit one to adopt the t'Hooft-Passarino-Veltmann formulation directly.

We circumvent these difficulties by carrying out the computation with relativistic baryon propagators and expanding our result in powers of $p / M$. Doing so allows us to evaluate the loop integrals using the standard formulation of Refs. $[23,24]$. It has been shown in other contexts [25] that doing so allows one to recover the heavy baryon result so long as the external momenta are sufficiently small. Moreover, our loop results are entirely nonanalytic in $p$ and, thus, must match the corresponding nonanalytic results obtained with heavy baryon propagators. To the order of our analysis, there exist no four-fermion operators that could account for differences between relativistic and nonrelativistic treatments of $A_{n}$.

The one-loop $\mathcal{M}_{\gamma \gamma}$ is nominally infrared singular and must, therefore, be regulated with an IR regulator such as a photon mass. On general grounds, the regulator dependence should be canceled by a corresponding dependence of the bremsstrahlung contribution to the spin-dependent cross section. As is well known, such a cancellation occurs for an unpolarized scattering cross section. In Appendix B, we work out the corresponding bremsstrahlung contribution to $A_{n}$ and show that it vanishes identically. Consequently, Im $\mathcal{M}_{\gamma \gamma}$ must be IR regulator-independent.

In general, the amplitude $\mathcal{M}_{\gamma \gamma}$ depends on each of the eleven integrals obtained in Ref. [24]. The imaginary part, however, depends on only four,

$$
D_{0}=\frac{2 \pi}{-t} \ln \left(\frac{-t}{\lambda^{2}}\right) \frac{1}{\sqrt{\Lambda}} \Theta\left(s-(m+M)^{2}\right),
$$

$$
\begin{gathered}
C_{0}(1,2,3)=\frac{\pi}{\sqrt{\Lambda}} \ln \left(\frac{\Lambda}{s \lambda^{2}}\right) \Theta\left(s-(m+M)^{2}\right), \\
C_{0}(1,3,4)=C_{0}(1,2,3)=C_{0}, \\
B_{0}(1,3)=\pi \frac{\sqrt{\Lambda}}{s} \Theta\left(s-(m+M)^{2}\right),
\end{gathered}
$$

where the three labels associated with the $B_{0}$ and $C_{0}$ functions indicate which propagators are used for the two-point and three-point integral as discussed in Appendix $\mathrm{C}, \lambda$ is the photon mass, and

$$
\Lambda=s^{2}-2 s\left(M^{2}+m^{2}\right)+\left(M^{2}-m^{2}\right)^{2} .
$$

These integrals have been previously computed in Refs. [24,26] (in [26] they are obtained by the use of dispersion techniques). The $D_{0}$ and $C_{0}$ loop integrals diverge as $\lambda \rightarrow 0$, but the combination

$$
2 C_{0}+D_{0} t=\frac{2 \pi}{\sqrt{\Lambda}} \ln \left(\frac{\Lambda}{-s t}\right) \Theta\left(s-(m+M)^{2}\right)
$$

is finite in this limit and is the only combination of $D_{0}$ and $C_{0}$ integrals that is so. As such, the two-photon contribution to $A_{n}$ must only contain terms proportional to this combination or to the $B_{0}$ integral.

In evaluating the loop contributions to $A_{n}$, it is most efficient to identify the terms in $\mathcal{M}_{\gamma \gamma}$ that generate the correlation of Eq. (1) by carrying out the Dirac algebra in the interference term $\operatorname{Im} \mathcal{M}_{\gamma \gamma} \mathcal{M}_{\gamma}^{*}$ before evaluating the momentum integrals. After carrying out the momentum integration, the contribution from the box diagram of Fig. 1(a) is

$$
\begin{aligned}
2 \operatorname{Im} \mathcal{M}_{\gamma \gamma}^{\text {box }} \mathcal{M}_{\gamma}^{*}= & -\frac{(4 \pi \alpha)^{2}}{4 \pi^{4} t} \frac{16 m \pi^{2}(4 \pi \alpha)}{(\Lambda+s t)} \epsilon^{\mu \nu \alpha \beta} P_{\mu} S_{\nu} K_{\alpha} K_{\beta}^{\prime}\left\{\left[4\left(M^{2}-m^{2}-3 s\right) M^{2} R+\kappa\left\{(6 R+2) \Lambda-\left[\left(m^{2}-M^{2}-s\right) R+2 s\right] t\right\}\right.\right. \\
& +\kappa^{2} R \frac{1}{8 M^{2}(\Lambda+s t)}\left\{2\left(3 m^{3}+16 M^{2}\right) \Lambda^{2}+\Lambda\left[11 m^{4}-2\left(13 M^{2}+8 s\right) m^{2}+15 M^{4}+11 s^{2}+14 M^{2} s\right]\right. \\
& \left.\left.\left.\times t+4 s\left[2 m^{4}-\left(5 M^{2}+4 s\right) M^{2}+3 M^{4}+2 s^{2}-3 M^{2} s\right] t^{2}\right\}\right]\left(2 C_{0}+D_{0} t\right)-4 \frac{\Lambda+t s}{\Lambda}\left(\kappa^{2}+4 \kappa+2\right) B_{0}\right\} .
\end{aligned}
$$

Here, $s, t$, and $u$ are the Mandelstaam variables, $\kappa=\mu-1$ is the nucleon anomalous magnetic moment, and

$$
R-1=t\left[\frac{\kappa}{4 M^{2}}-\frac{C_{r}}{M^{2}}\right] .
$$

To obtain the result consistent with our power counting, we expand Eq. (8) in powers of $p / M$ up to second order relative to the leading term ${ }^{4}$,

\footnotetext{
${ }^{4}$ This procedure introduces no ambiguities because $\operatorname{Im} \mathcal{M}_{\gamma \gamma}$ is finite to the order of our analysis.
} 


$$
\begin{aligned}
\operatorname{Im} \mathcal{M}_{\gamma \gamma}^{\text {box }} \mathcal{M}_{\gamma}^{*}= & -\frac{(4 \pi \alpha)^{2}}{t} \frac{32 \pi^{2} \alpha m M}{\sqrt{E^{2}-m^{2}}\left(\left(E^{2}-m^{2}+t / 4\right)+\frac{E t}{2 M}+\frac{m^{2} t}{4 M^{2}}\right)} \epsilon^{\mu \nu \alpha \beta} P_{\mu} S_{\nu} K_{\alpha} K_{\beta}^{\prime} \\
& \times\left\{[ \operatorname { l n } ( \frac { 4 ( E ^ { 2 } - m ^ { 2 } ) } { - t } ) - 2 E / M + ( 2 E ^ { 2 } - m ^ { 2 } ) / M ^ { 2 } ] \left[R+\frac{3 E}{M}+\frac{2 m^{2}}{M^{2}}+\frac{\kappa^{2}}{M^{2}} \frac{32\left(E^{2}-m^{2}\right)^{2}+t^{2} / 2+10\left(E^{2}-m^{2}\right) t}{4\left(E^{2}-m^{2}\right)+t}\right.\right. \\
& \left.\left.+\frac{4 \kappa}{M^{2}}\left(m^{2}-E^{2}\right)\right]-\frac{\kappa^{2}+4 \kappa+2}{M^{2}}\left[\left(E^{2}-m^{2}\right)+\frac{t}{4}\right]\right\} \Theta\left(s-(m+M)^{2}\right)
\end{aligned}
$$

where the $\Theta$ function arises from the integrals $2 C_{0}+2 D_{0} t$ and $B_{0}$. Note that we have retained the $m$ dependence purely for illustrative purposes, as $m<<E$ for the experiments of interest here. The corresponding contribution from the crossed-box diagram can be obtained by crossing symmetry with the replacement $s \rightarrow u$. In this case, the $\Theta$ function vanishes, so only $\operatorname{Im} \mathcal{M}_{\gamma \gamma}^{\text {box }} \mathcal{M}_{\gamma}^{*}$ contributes.

In the expression (10), the terms that go as powers of $E / M$ or $m / M$ but do not contain factors of $\kappa$ or $C_{r}$ arise purely from recoil effects. The proton charge radius contributes solely via $\mathcal{M}_{\gamma}$. Although it also contributes to the absorptive part of $\mathcal{M}_{\gamma \gamma}$, the resulting terms do not contribute to the spin-dependent correlation of Eq. (1). Including the magnetic moment, charge radius, and recoil-order terms in $\mathcal{M}_{\gamma}$ along with the loop contributions in Eq. (10) leads to the following expression for the VAP:

$$
\begin{aligned}
A_{n}= & -\frac{2 \alpha t m}{\sqrt{E^{2}-m^{2}}\left(\left(E^{2}-m^{2}+t / 4\right)+\frac{E t}{2 M}+\frac{m^{2} t}{4 M^{2}}\right)} \vec{S} \cdot \vec{K} \times \vec{K}^{\prime}\left\{\left[\ln \left(\frac{4\left(E^{2}-m^{2}\right)}{-t}\right)-2 E / M+\left(2 E^{2}-m^{2}\right) / M^{2}\right]\right. \\
& \left.\times\left[R+\frac{3 E}{M}+\frac{2 m^{2}}{M^{2}}+\frac{\kappa^{2}}{M^{2}} \frac{32\left(E^{2}-m^{2}\right)^{2}+t^{2} / 2+10\left(E^{2}-m^{2}\right) t}{4\left(E^{2}-m^{2}\right)+t}+\frac{4 \kappa}{M^{2}}\left(m^{2}-E^{2}\right)\right]-\frac{\kappa^{2}+4 \kappa+2}{M^{2}}\left[\left(E^{2}-m^{2}\right)+\frac{t}{4}\right]\right\} \\
& \times\left[\left(8 E^{2}+2 t\right) R^{2}+\frac{4 E t}{M}+t \frac{t+2 m^{2}+2 \kappa\left(t+2 m^{2}\right)+\kappa^{2}\left[t+4\left(m^{2}-E^{2}\right)\right] / 2}{M^{2}}\right]^{-1} .
\end{aligned}
$$

Dropping all terms that go as powers of $E / M, m / M$, or $t / M^{2}$ yields the result obtained in Ref. [11] that was obtained for scattering from an infinitely heavy, pointlike proton.

\section{RESULTS AND DISCUSSION}

The expression for $A_{n}$ given in Eq. (11) provides a parameter-free prediction for low-energy electron scattering. In Figs. 4 and 5, we plot $A_{n}$ as a function of energy for fixed laboratory frame scattering angles $\theta=146.1^{\circ}$ (Fig. 4) and $\theta$ $=30^{\circ}$ (Fig. 5), while in Fig. 6 we show the VAP for fixed energy $E=192 \mathrm{MeV}$ while varying $\theta$. In call cases, the leading-order calculation is shown for comparison. In Fig. 6, the relative importance of the recoil, magnetic moment, and charge radius contributions is also indicated.

The result obtained in the SAMPLE measurement is also shown. While the leading-order calculation overestimates the magnitude of $A_{n}$ by a factor of roughly 4 , inclusion of the higher-order terms considered here produces agreement with the experimental value. Interestingly, there appears to be scant evidence that dynamical pions or the $\Delta$ play a significant role in $A_{n}$ for this kinematic region $(E=192 \mathrm{MeV})$, despite one's expectation that they might.

At higher energies, our result for $A_{n}$ cannot be considered reliable, since the convergence of the effective theory expansion breaks down for $E \sim M$. The A4 Collaboration at Mainz has measured $A_{n}$ at $E=570.3 \mathrm{MeV}$ and $E=854.3 \mathrm{MeV}$ and $25^{\circ} \leqslant \theta \leqslant 35^{\circ}$. Preliminary results for the higher-energy VAP have been reported in Ref. [15]. A comparison with our computation indicates that the preliminary experimental values

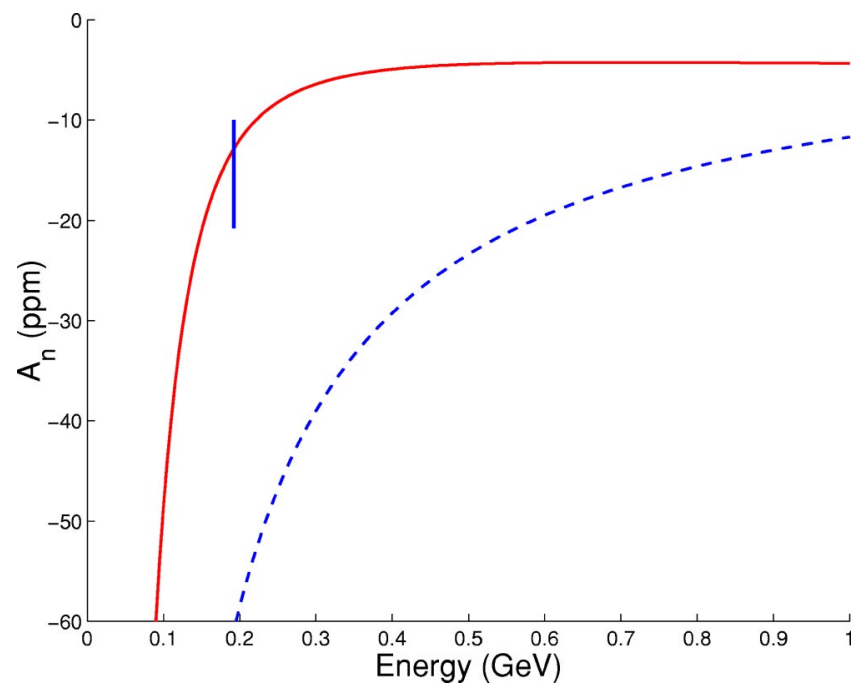

FIG. 4. (Color online) VAP vs energy for fixed scattering angle, $\theta=146.1^{\circ}$. The dashed line is the leading-order result, and the solid line shows the full calculation. The SAMPLE result [10] is also shown at $E=192 \mathrm{MeV}$. 


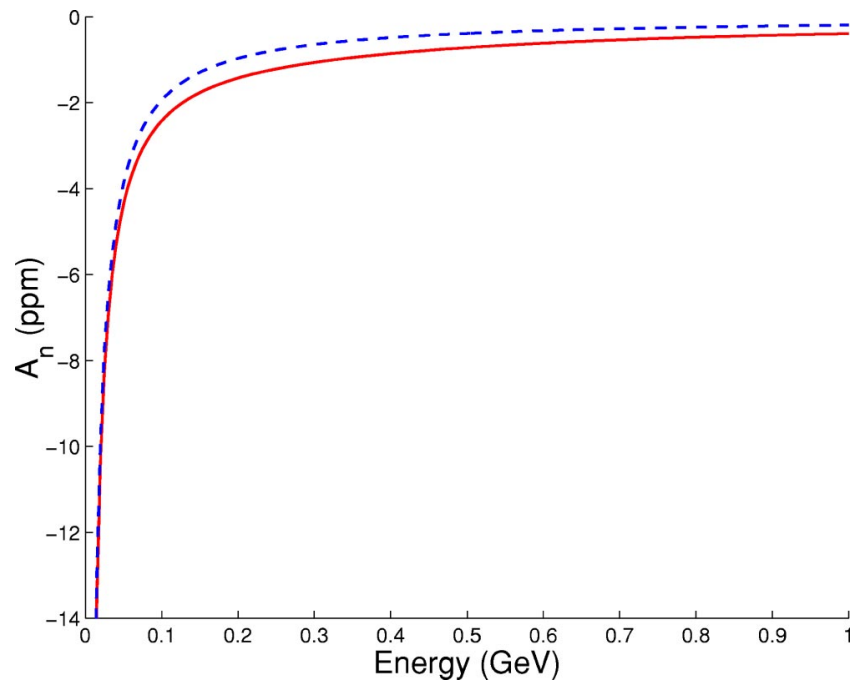

FIG. 5. (Color online) VAP vs energy for fixed scattering angle, $\theta=30^{\circ}$. The dashed line is the leading-order result, and the solid line shows the full calculation.

for forward angle scattering and higher energies are substantially larger in magnitude than we are able to obtain via the low-energy expansion to $O(E / M)^{2}$. Presumably, a resummation of higher-order contributions in $E / M$ using nonperturbative techniques, such as dispersion relations, would be required to compute reliably $A_{n}$ in this domain [12,27-30]. We would also expect that inclusion of nucleon resonances ${ }^{5}$ and pions as explicit degrees of freedom would be needed to account for the experimental results.

One indication of the possible strength of these higherorder contributions may be given by considering the T-odd, P-even dimension-7 operators. As shown in Appendix B, there exist two $d=7$ operators that could, in principle, contribute. From an explicit calculation, we find that only one of the two $-O_{e N}^{7 a}$-leads to a nonvanishing $A_{n}$. Here, it is useful to consider the form of this operator for relativistic proton fields, $N$,

$$
O_{e N}^{7 a}=\frac{\alpha^{2} C_{7 a}}{M^{3}} \bar{e} \sigma^{\mu \nu} \gamma_{5}(\vec{D}+\stackrel{\leftarrow}{D})_{\nu} e \bar{N} \gamma_{5} \gamma_{\mu} N
$$

Rewriting this operator in terms of the heavy fields $B_{v}$ leads to

$$
\tilde{O}_{e N}^{7 a}=-2 \frac{\alpha^{2} C_{7 a}}{M^{2}} \bar{e} \sigma^{\mu \nu} \gamma_{5}(\overleftarrow{D}+\vec{D})_{\nu} e \bar{B}_{v} S_{\mu}^{v} B_{v}
$$

where $S_{\mu}^{v}$ is the nucleon spin. The contribution from $\widetilde{O}_{e N}^{7 a}$ to the interference amplitude $\operatorname{Im} \tilde{\mathcal{M}}_{e N}^{7 a} \mathcal{M}_{\gamma}^{*}$ goes as $\epsilon^{\mu \nu \alpha \beta} S_{\mu} v_{\nu} v_{\alpha} K_{\beta}^{\prime}$ and, thus, vanishes. On the other hand, using the relativistic form of the operator, $O_{e N}^{7 a}$, leads to the

${ }^{5}$ For recent studies that pertain to such contributions, see Refs. [32-34].

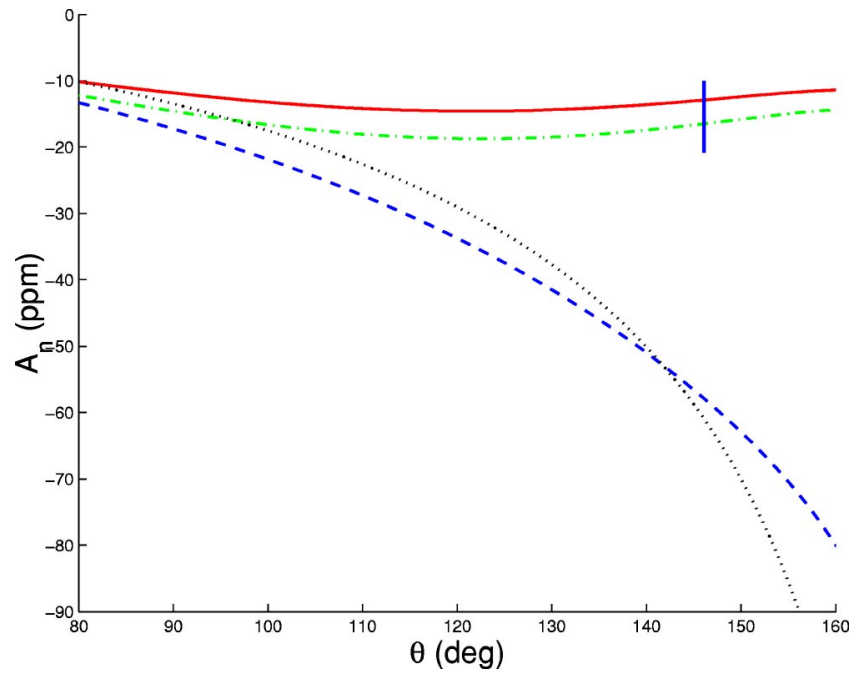

FIG. 6. (Color online) VAP vs scattering angle for the SAMPLE kinematics $(E=192 \mathrm{MeV})$. The dotted line gives the leading-order result, the dashed line adds the recoil corrections, the dash-dotted line adds the magnetic corrections, and the solid line shows the full calculation through $O(p / M)^{2}$.

correlation $\epsilon^{\mu \nu \alpha \beta} S_{\mu} P_{\nu} P_{\alpha}^{\prime} K_{\beta}^{\prime}$ that is nonvanishing for $P \neq P^{\prime}$. The resulting contribution to the VAP is

$$
A_{n}^{(7)}=\frac{\alpha C_{7 a}}{4 \pi} \frac{t^{2}|\vec{K}|\left|\vec{K}^{\prime}\right| \sin \theta}{M^{2}\left[8 M^{2} E^{2}+2(2 E+M) t M+t^{2}\right]},
$$

a result that is $O(p / M)^{4}$. In short, the only heavy baryon operators that can contribute involve either fields with two different velocities (viz., $B_{v}$ and $B_{v^{\prime}}$ ) whose contribution requires nonzero proton recoil, or dimension- 8 operators involving the $B_{v}$ fields only and carrying an additional $p / M$ recoil suppression.

The SAMPLE result for $A_{n}$ allows for a nonvanishing, but small coefficient for the leading, higher-order T-odd, P-even operator. Using the relativistic operator $O_{e N}^{7 a}$ for illustration and including the loop contributions through $O(p / M)^{2}$ leads to $C_{7 a}=3.07 \pm 6.64$. Naive dimensional analysis would have suggested a magnitude for $C_{7 a}$ or order unity, so the SAMPLE results do not appear to imply the presence of any unnatural hadronic scale physics. We may now use this range for $C_{7 a}$ to estimate the possible size of higher-order effects at other kinematics. The resulting band is shown in Fig. 7 for backward angles $\left(\theta=146.1^{\circ}\right)$ and in Fig. 8 for forward angles $\left(\theta=30^{\circ}\right)$. For the Mainz measurement at $E=570 \mathrm{MeV}$ and $\theta=30^{\circ}$, we find $-2.0 \leqslant A_{n}^{(7)} \leqslant 0.7 \mathrm{ppm}$, while $A_{n}^{\text {loop }}=$ $-0.64 \mathrm{ppm}$. Thus, one might expect the impact of the physics we have integrated out to grow in importance relative to the loop effects considered here as the energy of the beam is increased, and it appears reasonable to expect a magnitude of a few ppm at the Mainz kinematics. We caution, however, that the precise value obtained in our calculation is unlikely to be correct in this energy regime, where the convergence of the $E / M$ expansion is slow at best.

As a final comparison, we also consider $A_{n}$ in fixed target, polarized Møller scattering. The VAP for this process has 


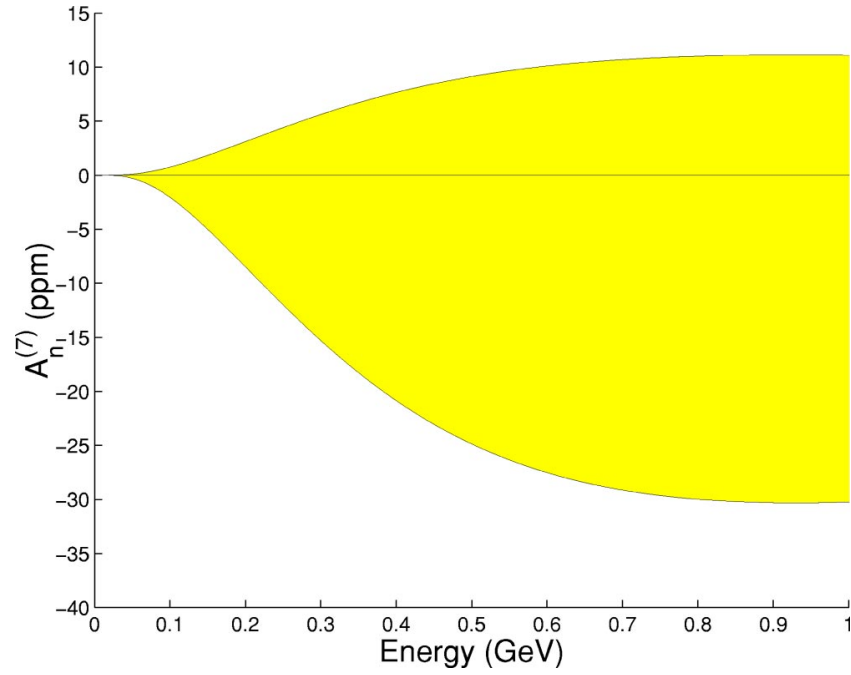

FIG. 7. (Color online) Possible contribution from the dimension seven, T-odd, P-even operator $O_{e N}^{7 a}$ to the backward angle VAP $(\theta$ $=146.1^{\circ}$ ).

been measured at SLAC by the E158 Collaboration [16], and one expects results to be forthcoming in the near future. Calculations of this quantity have been performed by several authors [17-19]. As a cross-check on our VAP for ep scattering, we carry out the analogous calculation here. It can be performed completely relativistically without performing an expansion in electron energy. However, since we are now dealing with identical particles in the final state, we need to compute the interference between tree diagams in Fig. 9(b) and the box diagrams of Fig. 9(a). For the SLAC measurement, one has $E=46 \mathrm{GeV}$. Performing the calculation in the center of mass frame, we obtain

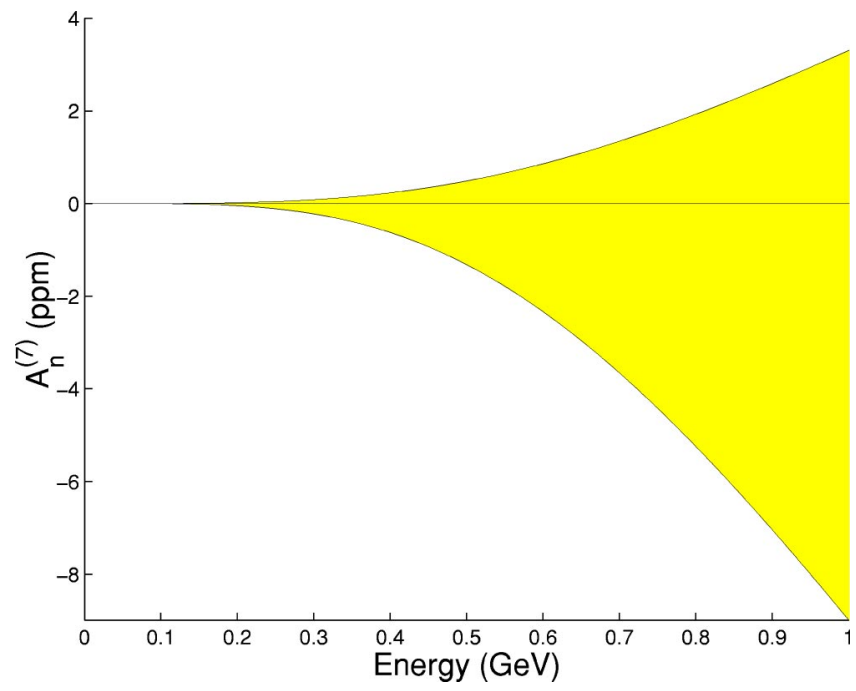

FIG. 8. (Color online) Possible contribution from $O_{e N}^{7 a}$ to the VAP at $\theta=30^{\circ}$, given constraints on the operator coefficient $C_{7 a}$ implied by the SAMPLE result.

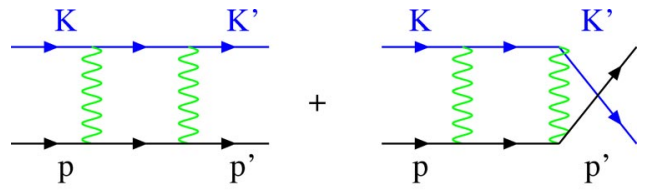

(a)
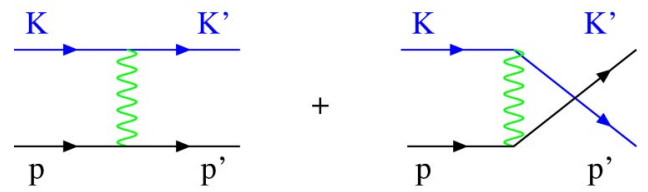

(b)

FIG. 9. (Color online) Diagrams contributing to the VAP for Møller scattering.

$$
\begin{aligned}
\frac{d \sigma^{\uparrow}}{d \Omega}-\frac{d \sigma^{\downarrow}}{d \Omega}= & \frac{\alpha^{3}}{8} \frac{m}{t^{2} u^{2} \sqrt{s}} \sin \theta \sqrt{1-\frac{4 m^{2}}{s}} \\
& \times\left\{3 ( s - 4 m ^ { 2 } ) \left[t\left(u-s+2 m^{2}\right) \ln \left(\frac{-t}{s-4 m^{2}}\right)\right.\right. \\
& \left.\left.-u\left(t-s+2 m^{2}\right) \ln \left(\frac{-u}{s-4 m^{2}}\right)\right]-2(t-u) t u\right\} \\
\frac{d \sigma^{\uparrow}}{d \Omega}+\frac{d \sigma^{\downarrow}}{d \Omega}= & \frac{\alpha^{2}}{2 s t^{2} u^{2}}\left[\left(t^{2}+t u+u^{2}\right)^{2}\right. \\
& \left.+4 m^{2}\left(m^{2}-t-u\right)\left(t^{2}-t u+u^{2}\right)\right],
\end{aligned}
$$

Our results are in agreement with those of Refs. [17-19]. ${ }^{6}$ The resulting asymmetry is ploted in Fig. 10, and agrees with the corresponding figure in Ref. [19] (note thatin Ref. [19], the VAP is plotted versus $\cos \theta$ rather than versus $\theta$ as we do here).

\section{CONCLUSIONS}

In this study, we have computed the low-energy, backward angle VAP using an effective theory involving electrons, photons, and protons, and we have obtained a parameter-free prediction through $O(p / M)^{2}$. The VAP to this order is determined entirely by the imaginary part of the interference between the two-photon exchange, one-loop amplitude, and the tree-level one-photon-exchange amplitude. In the limit that $M \rightarrow \infty$, our result exactly reproduces the VAP obtained in Ref. [11] for scattering from a structureless, infinitely heavy proton that over predicts the magnitude of $A_{n}$ at the kinematics of the SAMPLE experiment. We find that inclusion of all contributions through $O(p / M)^{2}$ leads to

\footnotetext{
${ }^{6}$ In Ref. [19], $O\left(\alpha^{2}\right)$ contributions arising from initial and final state radiation effects were also computed. The corresponding contributions for the $e p$ VAP are smaller than the hadronic uncertainties arising at $O(\alpha)$, so we do not consider them.
} 


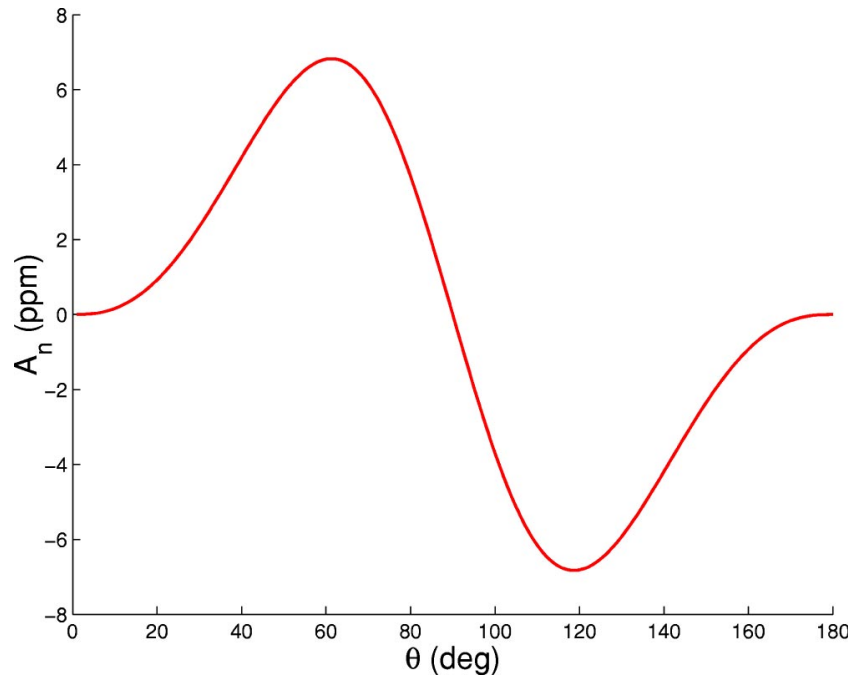

FIG. 10. (Color online) The Møller VAP vs CM scattering angle at the E158 kinematics.

agreement with experiment and leaves little room for important effects arising from dynamical pions or nucleon resonances at these energies. The leading counterterm contributions arise at $O(p / M)^{4}$ and are consistent with zero. Thus, the SAMPLE measurement provides no evidence for unusual hadronic physics effects at these scales. The data also constrain the magnitude of the counterterm coefficients to be of natural size, and lead one to expect the VAP as measured by the A4 Collaboration at Mainz to be at most of the order of a few ppm. Given the range of validity of our effective theory, however, we cannot produce a reliable prediction for VAP at the Mainz energies.

In this context, the results of the SAMPLE measurement have notable consequences for studies of weak interaction processes. In the case of both neutron $\beta$-decay and parityviolating ep scattering, theoretical consideration of final state QED corrections to the leading-order weak amplitudes is important for the interpretation of various measurements [13]. To the extent that these measurements involve relatively low lepton energies, an analogous effective field theory computation of one-loop graphs involving the exchange of one weak vector boson and one photon should be reliable at the $\sim 20 \%$ level relative to the size of other $O(\alpha)$ corrections. Future, more precise measurements of the VAP at low energies and overa range of angles would provide important tests of this provisional assessment.

One might also ask how competitive the SAMPLE measurement is with other direct searches for new T-odd, P-even interactions. As discussed in Refs. [4,5], direct searches are most relevant in symmetry-breaking scenarios wherein parity is broken at or above the scale for the breakdown of T. Existing direct searches imply that $\alpha_{T} \lesssim$ few $\times 10^{-3}$, where $\alpha_{T}$ is the ratio of a typical T-odd, P-even nuclear matrix element to those of the residual strong interaction. When translated into bounds on generic, dimension-7 operator coefficients $C_{7}$ [under the normalization of Eq. (12)], one obtains $\left|C_{7}\right| \lesssim 2$. The sensitivity of the SAMPLE measurement is comparable. Given that conventional, hadronic final state effects that have been integrated out in our computation naturally imply a value of $C_{7 a}$ with a magnitude of order unity, it appears unlikely that one will be able to circumvent the corresponding theoretical hadronic uncertainties as needed to make the VAP a direct probe of new physics. On the other hand, lowenergy studies of $A_{n}$ could provide important information for the theoretical interpretation of other precision, electroweak observables.

\section{ACKNOWLEDGMENTS}

It is a pleasure to thank C. Bauer, V. Cirigliano, B. Holstein, T. Ito, A. Kurylov, Y. Kolomensky, K. Kumar, F. Maas, D. Mack, C. Maekawa, R. D. McKeown, G. Prezeau, P.A. Souder, U. van Kolck, P. Vogel, and S.P. Wells for useful discussions. This work was supported in part under DOE Contract No. DE-FG03-88ER40397 and NSF Grant No. PHY-0071856.

\section{APPENDIX A: BREMSSTRAHLUNG COMPUTATION}

Here, we show that the bremsstrahlung amplitudes corresponding to Fig. 2 give a vanishing contribution to the VAP. The amplitudes are

$$
\begin{aligned}
\mathcal{M}^{a}= & \frac{-i}{q^{2}} \bar{u}\left(K^{\prime}\right)(i e) \gamma_{\mu} \frac{i(\mathbb{K}-l)+m}{(K-l)^{2}-m^{2}}(i e) \\
& \times \gamma^{\alpha} \epsilon_{\alpha} \frac{1+\gamma_{5} \$}{2} u(K) \bar{u}\left(p^{\prime}\right)(i e) \gamma^{\mu} u(p),
\end{aligned}
$$

$$
\begin{aligned}
\mathcal{M}^{b}= & \frac{-i}{q^{2}} \bar{u}\left(K^{\prime}\right)(i e) \gamma^{\alpha} \epsilon_{\alpha} \frac{i\left(K^{\prime}+l\right)+m}{\left(K^{\prime}+l\right)^{2}-m^{2}}(i e) \gamma_{\mu} \frac{1+\gamma_{5} \$}{2} \\
& \times u(K) \bar{u}\left(p^{\prime}\right)(i e) \gamma_{\mu} u(p)
\end{aligned}
$$

$$
\begin{aligned}
\mathcal{M}^{c}= & \frac{-i}{q^{2}} \bar{u}\left(K^{\prime}\right)(i e) \gamma^{\mu} u(K) \bar{u}\left(p^{\prime}\right)(i e) \gamma^{\mu} \\
& \times \frac{i\left(p^{\prime}+l\right)+M}{\left(p^{\prime}+l\right)^{2}-M^{2}}(i e) \gamma^{\alpha} \epsilon_{\alpha} u(p), \\
\mathcal{M}^{d}= & \frac{-i}{q^{2}} \bar{u}\left(K^{\prime}\right)(i e) \gamma^{\mu} u(K) \bar{u}\left(p^{\prime}\right)(i e) \gamma^{\alpha} \epsilon_{\alpha} \\
& \times \frac{i(p-l)+M}{(p-l)^{2}-M^{2}}(i e) \gamma^{\mu} u(p) .
\end{aligned}
$$

Here, $l_{\mu}$ is the radiated photon momentum. The square of the invariant amplitude

$$
\mathcal{M}^{B}=\left|\mathcal{M}^{a}+\cdots+\mathcal{M}^{d}\right|^{2}
$$


depends on ten different products of leptonic and hadronic tensors. The leptonic tensors are

$$
\begin{aligned}
& L_{\mu \nu}^{a a}=\operatorname{Tr}\left(\left(\mathbb{K}^{\prime}+m\right) \gamma_{\mu} \frac{(\mathbb{K}-l+m)}{(K-l)^{2}-m^{2}} \gamma_{\alpha} \frac{1+\gamma_{5} \not}{2}(\mathbb{K}+m)\right. \\
& \left.\times \gamma_{\beta} \frac{(K-l+m)}{(K-l)^{2}-m^{2}} \gamma_{\nu}\right) \epsilon^{\alpha} \epsilon^{* \beta}, \\
& L_{\mu \nu}^{a b}=\operatorname{Tr}\left(\left(\mathbb{K}^{\prime}+m\right) \gamma_{\mu} \frac{(\mathbb{K}-l+m)}{(K-l)^{2}-m^{2}} \gamma_{\alpha} \frac{1+\gamma_{5} \mathbb{S}}{2}(\mathbb{K}+m)\right. \\
& \left.\times \gamma_{\nu} \frac{\left(\mathbb{K}^{\prime}+l+m\right)}{\left(K^{\prime}+l\right)^{2}-m^{2}} \gamma_{\beta}\right) \epsilon^{\alpha} \epsilon^{* \beta}, \\
& L_{\mu \nu}^{a c}=\operatorname{Tr}\left(\left(\mathbb{K}^{\prime}+m\right) \gamma_{\mu} \frac{(\mathbb{K}-l+m)}{(K-l)^{2}-m^{2}} \gamma_{\alpha} \frac{1+\gamma_{5} \$}{2}(\mathbb{K}+m) \gamma_{\nu}\right) \boldsymbol{\epsilon}^{\alpha}, \\
& L_{\mu \nu}^{a d}=L_{\mu \nu}^{a c}, \\
& L_{\mu \nu}^{b b}=\operatorname{Tr}\left(\left(\mathbb{K}^{\prime}+m\right) \gamma_{\alpha} \frac{\left(\mathbb{K}^{\prime}+l+m\right)}{\left(K^{\prime}+l\right)^{2}-m^{2}} \gamma_{\mu} \frac{1+\gamma_{5} \$}{2}(\mathbb{K}+m)\right. \\
& \left.\times \gamma_{\nu} \frac{\left(\mathbb{K}^{\prime}+l+m\right)}{\left(K^{\prime}+l\right)^{2}-m^{2}} \gamma_{\beta}\right) \epsilon^{\alpha} \epsilon^{* \beta}, \\
& L_{\mu \nu}^{b c}=\operatorname{Tr}\left(\left(\mathbb{K}^{\prime}+m\right) \gamma_{\alpha} \frac{\left(\mathbb{K}^{\prime}+l+m\right)}{\left(K^{\prime}+l\right)^{2}-m^{2}} \gamma_{\mu} \frac{1+\gamma_{5} \not}{2}\right. \\
& \left.\times(\mathbb{K}+m) \gamma_{\nu}\right) \boldsymbol{\epsilon}^{\alpha} \\
& L_{\mu \nu}^{b d}=L_{\mu \nu}^{b c} \\
& L_{\mu \nu}^{c c}=\operatorname{Tr}\left(\left(\mathbb{K}^{\prime}+m\right) \gamma_{\mu} \frac{1+\gamma_{5} \$}{2}(\mathbb{K}+m) \gamma_{\nu}\right) \text {, } \\
& L_{\mu \nu}^{c d}=L_{\mu \nu}^{c c} \\
& L_{\mu \nu}^{d d}=L_{\mu \nu}^{c c} .
\end{aligned}
$$

The corresponding hadronic tensors are

$$
\begin{gathered}
H_{a a}^{\mu \nu}=\operatorname{Tr}\left[\left(\not p^{\prime}+M\right) \gamma^{\mu}(\not p+M) \gamma^{\nu}\right], \\
H_{a b}^{\mu \nu}=H_{a a}^{\mu \nu}, \\
H_{a c}^{\mu \nu}=\operatorname{Tr}\left(\left(\not p^{\prime}+M\right) \gamma^{\mu}(\not p+M) \gamma^{\beta} \frac{(\not p-l+M)}{(p-l)^{2}-M^{2}} \gamma^{\nu}\right) \epsilon_{\beta}^{*}, \\
H_{a d}^{\mu \nu}=\operatorname{Tr}\left(\left(\not p^{\prime}+M\right) \gamma^{\mu}(\not p+M) \gamma^{\nu} \frac{\left(\not p^{\prime}+l+M\right)}{\left(p^{\prime}+l\right)^{2}-M^{2}} \gamma^{\beta}\right) \epsilon_{\beta}^{*}, \\
H_{b b}^{\mu \nu}=H_{a a}^{\mu \nu}, \\
H_{b c}^{\mu \nu}=H_{a a}^{\mu \nu},
\end{gathered}
$$

$$
\begin{aligned}
& H_{b d}^{\mu \nu}=H_{a d}^{\mu \nu}, \\
& H_{c c}^{\mu \nu}=\operatorname{Tr}\left(\left(p^{\prime}+M\right) \gamma^{\alpha} \frac{(p-l+M)}{(p-l)^{2}-M^{2}} \gamma^{\mu}(\not p+M)\right. \\
&\left.\times \gamma^{\nu} \frac{(\not p-l+M)}{(p-l)^{2}-M^{2}} \gamma^{\beta}\right) \epsilon_{\alpha} \epsilon_{\beta}^{*}, \\
& H_{c d}^{\mu \nu}= \operatorname{Tr}\left(\left(p^{\prime}+M\right) \gamma^{\alpha} \frac{(\not p-l+M)}{(p-l)^{2}-M^{2}} \gamma^{\mu}(\not p+M)\right. \\
&\left.\times \gamma^{\beta} \frac{\left(\not p^{\prime}+l+M\right)}{\left(p^{\prime}+l\right)^{2}-M^{2}} \gamma^{\nu}\right) \epsilon_{\alpha} \epsilon_{\beta}^{*}, \\
& H_{d d}^{\mu \nu}= \operatorname{Tr}\left(\left(\not p^{\prime}+M\right) \gamma^{\mu} \frac{\left(\not b^{\prime}+l+M\right)}{\left(p^{\prime}+l\right)^{2}-M^{2}} \gamma^{\alpha}(\not p+M)\right. \\
&\left.\times \gamma^{\beta} \frac{\left(\not p^{\prime}+l+M\right)}{\left(p^{\prime}+l\right)^{2}-M^{2}} \gamma^{\nu}\right) \epsilon_{\alpha} \epsilon_{\beta}^{*} .
\end{aligned}
$$

We now need to compute

$$
\begin{aligned}
\mathcal{M}^{B}=\sum_{\mathrm{pol}} \int d^{4} l\left\{\frac { 1 } { q ^ { 4 } } \left[L_{\mu \nu}^{a a} H_{a a}^{\mu \nu}+L_{\mu \nu}^{a b} H_{a b}^{\mu \nu}+L^{a c} H_{a c}^{\mu \nu}+L_{\mu \nu}^{a d} H_{a d}^{\mu \nu}\right.\right. \\
+L_{\mu \nu}^{b b} H_{b b}^{\mu \nu}+L_{\mu \nu}^{b c} H_{b c}^{\mu \nu}+L_{\mu \nu}^{b d} H_{b d}^{\mu \nu}+L_{\mu \nu}^{c c} H_{c c}^{\mu \nu} \\
\left.\left.+L_{\mu \nu}^{c d} H_{c d}^{\mu \nu}+L_{\mu \nu}^{d d} H_{d d}^{\mu \nu}\right]+\mathrm{H} . \mathrm{c} .\right\} \\
=\sum_{\mathrm{pol}} \int d^{4} l\left\{\frac { 1 } { q ^ { 4 } } \left[\left(H_{a c}^{\mu \nu}+H_{a d}^{\mu \nu}\right)\left(L_{\mu \nu}^{a c}+L_{\mu \nu}^{a d}\right)+H_{a a}^{\mu \nu}\left(L_{\mu \nu}^{a a}\right.\right.\right. \\
\left.\left.\left.\quad+L_{\mu \nu}^{a b}+L_{\mu \nu}^{b b}\right)+L_{c c}^{\mu \nu}\left(H_{\mu \nu}^{c c}+H_{\mu \nu}^{c d}+H_{\mu \nu}^{d d}\right)\right]+\mathrm{H} . \mathrm{c} .\right\}
\end{aligned}
$$

where the sum is over all polarizations of the radiated photon. We are only interested in the terms proportional to $\epsilon_{\alpha \beta \gamma \delta} S^{\alpha} k^{\beta} k^{\prime \gamma} p^{\delta}$. First we investigate the momentum integrals,

$$
\begin{aligned}
i \pi^{2} \mathcal{I}^{\mathcal{B}}= & \int d^{4} l\left[\frac{1}{\left(p^{\prime}+l\right)^{2}-M^{2}} \frac{1}{\left(p^{\prime}+l\right)^{2}-M^{2}}\right. \\
& +\frac{1}{\left(p^{\prime}+l\right)^{2}-M^{2}} \frac{1}{(p-l)^{2}-M^{2}} \\
& +\frac{1}{(p-l)^{2}-M^{2}} \frac{1}{(p-l)^{2}-M^{2}} \\
& \left.+\frac{1}{\left(k^{\prime}+l\right)^{2}-m^{2}} \frac{1}{\left(k^{\prime}+l\right)^{2}-m^{2}}+\cdots\right] .
\end{aligned}
$$

We can evaluate the generic two-point integral as defined by 


$$
\begin{aligned}
i \pi^{2} B\left(p^{2} ; m_{1}^{2}, m_{2}^{2}\right)= & \mu^{4-n} \int d^{n} q\left[\frac{1}{q^{2}+m_{1}^{2}-i \epsilon}\right. \\
& \left.\times \frac{1}{(q+p)^{2}+m_{2}^{2}-i \epsilon}\right] .
\end{aligned}
$$

We are only interested in the imaginary part of $B$. We find that above the physical threshold $s=-p^{2} \geqslant\left(m_{1}+m_{2}\right)^{2}$ this integral develops an imaginary part [31]

$$
\operatorname{Im} B\left(p^{2} ; m_{1}^{2}, m_{2}^{2}\right)=\pi \frac{\sqrt{\lambda\left(s, m_{1}^{2}, m_{2}^{2}\right)}}{s} \Theta\left(s-\left(m_{1}+m_{2}\right)^{2}\right) .
$$

Evaluating the $B$ functions for the kinematics involved here, we find that none of the integrals of Eq. (A6) develop an imaginary part. As such, evaluating the traces and performing the integration, we obtain a result of the form

$$
\begin{aligned}
\mathcal{M}^{\mathcal{B}}= & f_{1}(m, M, s, t, u)+f_{2}(m, M, s, t, u) \\
& \times i \epsilon_{\alpha \beta \gamma \delta} S^{\alpha} k^{\beta} k^{\prime}{ }_{p} p^{\delta}+\mathrm{H} . \mathrm{c} . \\
= & 2 f_{1}(m, M, s, t, u) .
\end{aligned}
$$

Hence, we find no contribution to $A_{n}$.

\section{APPENDIX B: LOCAL OPERATORS}

As discussed in the text, we are interested in computing the contribution to the VAP from local, four-fermion eeNN operators. The lowest dimension operators of this form have dimension 6 . First, we show by explicit calculation that all $d=6$ operators give vanishing contributions to $A_{n}$. The most general forms for the $d=6$ operators are

$$
\begin{gathered}
O_{e N}^{6 a}=\frac{\alpha^{2}}{M^{2}} \bar{e}\left(C_{1}+C_{2} \gamma_{5}\right) e \bar{N}\left(C_{1}^{\prime}+C_{2}^{\prime} \gamma_{5}\right) N, \\
O_{e N}^{6 b}=\frac{\alpha^{2}}{M^{2}} \bar{e}\left(C_{3}+C_{4} \gamma_{5}\right) \gamma^{\mu} e \bar{N}\left(C_{3}^{\prime}+C_{4}^{\prime} \gamma_{5}\right) \gamma^{\mu}, \\
O_{e N}^{6 c}=\frac{\alpha^{2}}{M^{2}} \bar{e}\left(C_{5}+C_{6} \gamma_{5}\right) \sigma^{\mu \nu} e \bar{N}\left(C_{5}^{\prime}+C_{6}^{\prime} \gamma_{5}\right) \sigma_{\mu \nu} N,
\end{gathered}
$$

where we have used relativistic nucleon fields $N$ (the corresponding argument carries over straightforwardly in the heavy baryon formalism). To make the above Hermitian we require all the constants $C_{e N}^{i}$ to be real. We now compute the interference of the amplitudes associated with these operators and the tree amplitude $\mathcal{M}_{\gamma}$, retaining only the desired structure $\epsilon_{\alpha \beta \gamma \delta} S^{\alpha} p^{\beta} K^{\gamma} K^{\prime \delta}$. The corresponding leptonic and hadronic tensors are

$$
\begin{gathered}
L_{6 a}^{\mu}=\operatorname{Tr}\left(\left(\mathbb{K}^{\prime}+m\right)\left(C_{1}+C_{2} \gamma_{5}\right) \frac{1+\gamma_{5} \$}{2}(\mathbb{K}+m) \gamma^{\mu}\right), \\
L_{6 b}^{\mu \nu}=\operatorname{Tr}\left(\left(\mathbb{K}^{\prime}+m\right)\left(C_{3}+C_{4} \gamma_{5}\right) \gamma^{\nu} \frac{1+\gamma_{5} \$}{2}(\mathbb{K}+m) \gamma^{\mu}\right), \\
L_{6 c}^{\mu \nu \alpha}=\operatorname{Tr}\left(\left(\mathbb{K}^{\prime}+m\right)\left(C_{5}+C_{6} \gamma_{5}\right) \sigma^{\nu \alpha} \frac{1+\gamma_{5} \$}{2}(\mathbb{K}+m) \gamma^{\mu}\right),
\end{gathered}
$$

$$
\begin{gathered}
H_{6 a}^{\mu}=\operatorname{Tr}\left(\left(\not p^{\prime}+m\right)\left(C_{1}^{\prime}+C_{2}^{\prime} \gamma_{5}\right)(\not p+m) \gamma^{\mu}\right), \\
H_{6 b}^{\mu \nu}=\operatorname{Tr}\left(\left(\not p^{\prime}+m\right)\left(C_{3}^{\prime}+C_{4}^{\prime} \gamma_{5}\right) \gamma^{\mu}(\not p+m) \gamma^{\mu}\right), \\
H_{6 c}^{\mu \nu \alpha}=\operatorname{Tr}\left(\left(\not p^{\prime}+m\right)\left(C_{5}^{\prime}+C_{6}^{\prime} \gamma_{5}\right) \sigma^{\mu \alpha}(\not p+m) \gamma^{\mu}\right), \\
\mathcal{M}_{6} \mathcal{M}_{\gamma}^{*}+\text { H.c. }= \\
+\frac{(4 \pi \alpha) \alpha^{2}}{t M^{2}}\left[L_{6 a}^{\mu} H_{\mu(6 a)}+L_{6 b}^{\mu \nu} H_{\mu \nu(6 b)}\right. \\
\left.+L_{6 c}^{\mu \nu \alpha} H_{\mu \nu \alpha(6 c)}\right]+ \text { H.c. }
\end{gathered}
$$

Evaluating the traces and keeping only the terms of interest, we obtain

$$
\begin{aligned}
\mathcal{M}_{6} \mathcal{M}_{\gamma}^{*}+\text { H.c. }= & i 16 \frac{(4 \pi \alpha) \alpha^{2}}{t M^{2}}\left(C_{1} C_{1}^{\prime} M-C_{4} C_{4}^{\prime} m\right) \epsilon_{\alpha \beta \gamma \delta} \\
& \times S^{\alpha} p^{\beta} K^{\gamma} K^{\prime \delta}+\text { H.c. }
\end{aligned}
$$

Since all the $C$ 's are real, we see there is no contribution from dimension- 6 terms. This results is as expected, as the operators $O_{6 a-c}$ are even under both T and $\mathrm{P}$.

Now consider $d=7$ operators. As for the $d=6$ operators, all contributions from T-even P-even $d=7$ operators will vanish. We may, however, write down two Hermitian T-odd, P-even $d=7$ operators,

$$
\begin{aligned}
O_{e N}^{7 a} & =\frac{\alpha^{2}}{M^{3}} C_{7 a} \bar{e} \gamma_{5} \sigma^{\mu \nu}(\stackrel{\leftarrow}{D}+\vec{D})_{\nu} e \bar{N} \gamma_{5} \gamma \mu N, \\
O_{e N}^{7 b} & =\frac{\alpha^{2}}{M^{3}} C_{7 b} \bar{e} \gamma_{5} \gamma_{\mu} e \bar{N} \gamma_{5} \sigma^{\mu \nu}(\stackrel{\leftarrow}{D}+\vec{D})_{\nu} N
\end{aligned}
$$

As before, we evaluate the interference of the above with $\mathcal{M}_{\gamma}$. The corresponding leptonic and hadronic tensors are

$$
\begin{gathered}
L_{7 a}^{\mu \nu}=i \operatorname{Tr}\left(\left(\mathbb{K}^{\prime}+m\right) \gamma_{5} \sigma^{\mu \alpha} q_{\alpha} \frac{1+\gamma_{5} \$}{2}(\mathbb{K}+m) \gamma^{\nu}\right) \\
L_{7 b}^{\mu \nu}=\operatorname{Tr}\left(\left(\mathbb{K}^{\prime}+m\right) \gamma_{5} \gamma^{\mu} \frac{1+\gamma_{5} \$}{2}(\mathbb{K}+m) \gamma^{\nu}\right) \\
H_{7 a}^{\mu \nu}=\operatorname{Tr}\left(\left(\not p^{\prime}+m\right) \gamma_{5} \gamma^{\mu}(\not p+m) \gamma^{\nu}\right) \\
H_{7 b}^{\mu \nu}=i \operatorname{Tr}\left(\left(p^{\prime}+m\right) \gamma_{5} \sigma^{\mu \alpha} q^{a}(\not p+m) \gamma^{\nu}\right) \\
\mathcal{M}_{7} \mathcal{M}_{\gamma}^{*}+\operatorname{H.c.}=i \frac{(4 \pi \alpha) \alpha^{2}}{t M^{3}}\left[C_{7 a} L_{7 a}^{\mu \nu} H_{\mu \nu(7 a)}\right. \\
\left.+C_{7 b} L_{7 b}^{\mu \nu} H_{\mu \nu(7 b)}\right]+ \text { H.c. }
\end{gathered}
$$

Evaluating the traces, we note that only the $L_{7 a}^{\mu \nu} H_{\mu \nu(7 a)}$ contributes,

$$
\mathcal{M}_{7} \mathcal{M}_{\gamma}+\text { H.c. }=\frac{16(4 \pi \alpha) \alpha^{2} C_{7 a}}{M^{3}} \epsilon_{\alpha \beta \gamma \delta} S^{\alpha} p^{\beta} k^{\gamma} k^{\prime \delta} .
$$

We are interested in the contribution such a term gives to the VAP. Keep only the leading piece of the tree amplitude, we get 


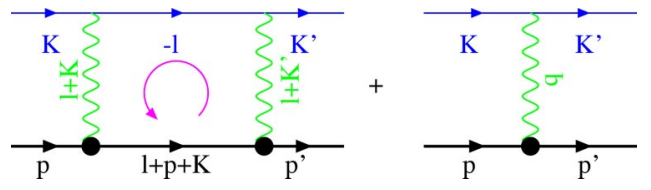

FIG. 11. (Color online) Momentum routing for the $\gamma \gamma$ box graph integrals.

$$
A_{n}^{(7)}=\frac{\alpha C_{7 a}}{4 \pi} \frac{t^{2}|\vec{K}|\left|\vec{K}^{\prime}\right| \sin \theta}{M^{2}\left[8 M^{2} E^{2}+2(2 E+M) t M+t^{2}\right]} .
$$

\section{APPENDIX C: LOOP INTEGRALS}

Here, we provide additional details about the computation of $\mathcal{M}_{\gamma \gamma}$. As noted in the text, the contribution from the crossed-box diagram vanishes, so we consider only $\operatorname{Im} \mathcal{M}_{\gamma \gamma}^{\text {box }} \mathcal{M}_{\gamma}^{*}$. Using the momentum routing shown in Fig. 11 we express the latter in terms of the leptonic and hadronic tensors:

$$
\begin{aligned}
L^{\mu \nu \alpha}= & \bar{u}\left(K^{\prime}\right)(i e) \gamma^{\mu} \frac{i(-t+m)}{l^{2}-m^{2}}(i e) \gamma^{\nu} \frac{1+\gamma_{5} \$}{2} u(K) \bar{u}(K) \\
& \times(i e) \gamma^{\alpha} u\left(K^{\prime}\right) \\
H_{\mu \nu \alpha}= & \bar{u}\left(p^{\prime}\right)\left(i e\left[1+r\left(l+K^{\prime}\right)^{2}\right] \gamma_{\mu}-\frac{\kappa \sigma_{\mu \beta}}{2 M}\left(l+K^{\prime}\right)^{\beta}\right) \\
& \times \frac{i\left(t+K^{\prime}+p^{\prime}+M\right)}{\left(l+K^{\prime}+p^{\prime}\right)^{2}-M^{2}}\left(i e\left[1+r(l+K)^{2}\right] \gamma_{\nu}\right. \\
& \left.+\frac{\kappa \sigma_{\nu \delta}}{2 M}(l+K)^{\delta}\right) u(p) \bar{u}(p)\left(i e\left[1+r\left(K-K^{\prime}\right)^{2}\right] \gamma_{\alpha}\right. \\
& \left.+\frac{\kappa \sigma_{\alpha \gamma}}{2 M}\left(K-K^{\prime}\right)^{\gamma}\right) u\left(p^{\prime}\right), \\
\mathcal{M}_{\gamma \gamma}^{\text {box }} \mathcal{M}_{\gamma}^{*} & =\int \frac{d^{4} l}{(2 \pi)^{2}} L^{\mu \nu \alpha} \frac{-i}{\left(l+K^{\prime}\right)^{2}} \frac{-i}{(l+K)^{2}} \frac{-i}{\left(K-K^{\prime}\right)^{2}} H_{\mu \nu \alpha},
\end{aligned}
$$

where

$$
r=R-1 \text {. }
$$

We define the loop integrals from above as follows:

$$
\begin{gathered}
i \pi^{2} D_{0}=\int d^{4} l \frac{1}{\left(l^{2}-m^{2}\right)\left(l+K^{\prime}\right)^{2}\left[\left(l+K^{\prime}+p^{\prime}\right)^{2}-M^{2}\right](l+K)^{2}}, \\
i \pi^{2} D_{\alpha}=\int d^{4} l \frac{l_{\alpha}}{\left(l^{2}-m^{2}\right)\left(l+K^{\prime}\right)^{2}\left[\left(l+K^{\prime}+p^{\prime}\right)^{2}-M^{2}\right](l+K)^{2}}, \\
i \pi^{2} D_{\alpha \beta} \\
\quad=\int d^{4} l \frac{l_{\alpha} l_{\beta}}{\left(l^{2}-m^{2}\right)\left(l+K^{\prime}\right)^{2}\left[\left(l+K^{\prime}+p^{\prime}\right)^{2}-M^{2}\right](l+K)^{2}},
\end{gathered}
$$

$$
\begin{aligned}
& i \pi^{2} D_{\alpha \beta \gamma} \\
& \quad=\int d^{4} l \frac{l_{\alpha} l_{\beta} l_{\gamma}}{\left(l^{2}-m^{2}\right)\left(l+K^{\prime}\right)^{2}\left[\left(l+K^{\prime}+p^{\prime}\right)^{2}-M^{2}\right](l+K)^{2}}, \\
& i \pi^{2} D_{\alpha \beta \gamma \delta} \\
& \quad=\int d^{4} l \frac{l_{\alpha} l_{\beta} l_{\gamma} l_{\delta}}{\left(l^{2}-m^{2}\right)\left(l+K^{\prime}\right)^{2}\left[\left(l+K^{\prime}+p^{\prime}\right)^{2}-M^{2}\right](l+K)^{2}} .
\end{aligned}
$$

In order to evaluate these integrals, we follow the methods of Refs. [23,24], and our notation follows that of Ref. [24]. To this end, we need to compute the following three-point functions:

$$
\begin{gathered}
i \pi^{2} C_{0}(1,2,3)=\int d^{4} l \frac{1}{\left(l^{2}-m^{2}\right)\left(l+K^{\prime}\right)^{2}\left[\left(l+K^{\prime}+p^{\prime}\right)^{2}-M^{2}\right]} \\
i \pi^{2} C_{0}(1,2,4)=\int d^{4} l \frac{1}{\left(l^{2}-m^{2}\right)\left(l+K^{\prime}\right)^{2}(l+K)^{2}}, \\
i \pi^{2} C_{0}(1,3,4)=\int d^{4} l \frac{1}{\left(l^{2}-m^{2}\right)\left[\left(l+K^{\prime}+p^{\prime}\right)^{2}-M^{2}\right](l+K)^{2}} \\
i \pi^{2} C_{0}(2,3,4)=\int d^{4} l \frac{1}{\left(l+K^{\prime}\right)^{2}\left[\left(l+K^{\prime}+p^{\prime}\right)^{2}-M^{2}\right](l+K)^{2}}
\end{gathered}
$$

and two-point functions

$$
\begin{gathered}
i \pi^{2} B_{0}(1,2)=\int d^{4} l \frac{1}{\left(l^{2}-m^{2}\right)\left(l+K^{\prime}\right)^{2}}, \\
i \pi^{2} B_{0}(1,3)=\int d^{4} l \frac{1}{\left(l^{2}-m^{2}\right)\left[\left(l+K^{\prime}+p^{\prime}\right)^{2}-M^{2}\right]},
\end{gathered}
$$

$$
i \pi^{2} B_{0}(1,4)=\int d^{4} l \frac{1}{\left(l^{2}-m^{2}\right)(l+K)^{2}},
$$

$$
i \pi^{2} B_{0}(2,4)=\int d^{4} l \frac{1}{\left(l+K^{\prime}\right)^{2}(l+K)^{2}},
$$

$$
\begin{aligned}
& i \pi^{2} B_{0}(2,3)=\int d^{4} l \frac{1}{\left(l+K^{\prime}\right)^{2}\left[\left(l+K^{\prime}+p^{\prime}\right)^{2}-M^{2}\right]}, \\
& i \pi^{2} B_{0}(3,4)=\int d^{4} l \frac{1}{\left[\left(l+K^{\prime}+p^{\prime}\right)^{2}-M^{2}\right](l+K)^{2}} .
\end{aligned}
$$

For all the $B, C$, and $D$ integrals above, we are interested only in the imaginary part. The only two-, three-, and fourpoint integrals with nonvanishing imaginary parts are 


$$
\begin{gathered}
\operatorname{Im} D_{0}=\frac{2 \pi}{-t} \ln \left(\frac{-t}{\lambda^{2}}\right) \frac{1}{\sqrt{\Lambda}} \Theta\left(s-(m+M)^{2}\right), \\
\operatorname{Im} C_{0}(1,2,3)=\frac{\pi}{\sqrt{\Lambda}} \ln \left(\frac{\Lambda}{s \lambda^{2}}\right) \Theta\left(s-(m+M)^{2}\right),
\end{gathered}
$$

$$
\operatorname{Im} C_{0}(1,3,4)=\operatorname{Im}\left[C_{0}(1,2,3)\right]=C_{0},
$$$$
\operatorname{Im} B_{0}(1,3)=\pi \frac{\sqrt{\Lambda}}{s} \Theta\left(s-(m+M)^{2}\right),
$$

In the above, $\lambda$ is the photon mass and $\Lambda=s^{2}-2 s\left(M^{2}+m^{2}\right)$ $+\left(M^{2}-m^{2}\right)^{2}$.

Although space considerations preclude a complete delineation of the calculation here, it is instructive to consider in more detail the evaluation of one of the four-point integrals required. Specifically, we consider

$$
D^{\alpha}=p_{1}^{\alpha} D_{11}+p_{2}^{\alpha} D_{12}+p_{3}^{\alpha} D_{13} .
$$

For the kinematics considered here, the Passarino and Veltman momenta and masses are

$$
\begin{gathered}
p_{1}=K, \quad m_{1}=m, \\
p_{2}=p, \quad m_{2}=0, \\
p_{3}=-p^{\prime}, \quad m_{3}=M, \\
p_{4}=-K^{\prime}, \quad m_{4}=0 .
\end{gathered}
$$

We then have for the $\operatorname{Im} D_{i j}$

$$
\operatorname{Im}\left(\begin{array}{l}
D_{11} \\
D_{12} \\
D_{13}
\end{array}\right)=X^{-1} \operatorname{Im}\left(\begin{array}{l}
R_{20} \\
R_{21} \\
R_{22}
\end{array}\right)
$$

where

$$
\begin{aligned}
R_{20} & =\frac{1}{2}\left[f_{1} D_{0}+C_{0}(1,3,4)-C_{0}(2,3,4)\right] \\
& =\frac{1}{2}\left(2 D_{0} m^{2}+C_{0}\right),
\end{aligned}
$$

$$
\begin{aligned}
R_{21} & =\frac{1}{2}\left[f_{2} D_{0}+C_{0}(1,2,4)-C_{0}(1,3,4)\right] \\
& =\frac{1}{2}\left[2 D_{0}\left(s-M^{2}-m^{2}\right)-C_{0}\right],
\end{aligned}
$$$$
R_{22}=\frac{1}{2}\left[f_{3} D_{0}+C_{0}(1,2,3)-C_{0}(1,2,4)\right]
$$$$
=\frac{1}{2}\left[-2 D_{0}\left(s-M^{2}-m^{2}\right)+C_{0}\right],
$$

where

$$
\begin{gathered}
f_{1}=m_{1}^{2}-m_{2}^{2}-p_{1}^{2}=2 m^{2}, \\
f_{2}=m_{1}^{2}-m_{2}^{2}+p_{1}^{2}-p_{5}^{2}=\left(s-M^{2}-m^{2}\right), \\
f_{3}=m_{2}^{2}-m_{4}^{2}-p_{4}^{2}+p_{5}^{2}=-f_{2},
\end{gathered}
$$

and where the inverse of the momentum matrix $X$ is

$$
X^{-1}=\left(\begin{array}{ccc}
p_{1}^{2} & p_{1} p_{2} & p_{1} p_{3} \\
p_{1} p_{2} & p_{2}^{2} & p_{2} p_{3} \\
p_{1} p_{3} & p_{2} p_{3} & p_{3}^{2}
\end{array}\right)^{-1}=\left(\begin{array}{ccc}
\frac{4 M^{2}-t}{\Lambda+t s} & \frac{3 M^{2}+m^{2}-s-t}{\Lambda+t s} & \frac{M^{2}-m^{2}+s}{\Lambda+t s} \\
\frac{3 M^{2}+m^{2}-s-t}{\Lambda+t s} & \frac{2\left(M^{2}+s+t\right) m^{2}-\left(s+t-M^{2}\right)^{2}-m^{4}}{t(\Lambda+t s)} & \frac{M^{2}-m^{2}}{\Lambda+t s}-\frac{1}{t} \\
\frac{M^{2}-m^{2}+s}{\Lambda+t s} & \frac{M^{2}-m^{2}}{\Lambda+t s}-\frac{1}{t} & \frac{s}{\Lambda+t s}-\frac{1}{t}
\end{array}\right) .
$$

After performing the necessary algebra, we obtain

$$
\begin{gathered}
\operatorname{Im}\left[D_{11}\right]=-\frac{D_{0}\left\{2\left[(m-M)^{2}-s\right]\left[(m+M)^{2}-s\right]+\left(m^{2}-M^{2}+s\right) t\right\}-2 C_{0}\left(s+M^{2}-m^{2}\right)}{2(\Lambda+t s)}, \\
\operatorname{Im}\left[D_{12}\right]=-\frac{D_{0}\left\{m^{4}+\left[t-2\left(M^{2}+s\right)\right]+\left(M^{2}-s\right)\left(M^{2}-s-t\right)\right\} m^{2}+2 C_{0}\left(m^{2}-M^{2}\right)}{2(\Lambda+t s)}, \\
\operatorname{Im}\left[D_{13}\right]=\frac{-D_{0} \Lambda+2 C_{0} s}{2(\Lambda+t s)} .
\end{gathered}
$$

Similar steps are required in evaluating the other four-point integrals. 
[1] emiT Collaboration, L. J. Lising et al., Phys. Rev. C 62, 055501 (2000).

[2] P. Herczeg, Time Reversal Violation in Nuclear Processes, in Symmetries and Fundamental Interactions in Nuclei, edited by W. C. Haxton and E. M. Henley (World Scientific, Singapore, 1995), p. 89 and references therein.

[3] F. Boehm, Time Reversal Tests in Nuclei, in Symmetries and Fundamental Interactions in Nuclei, edited by W. C. Haxton and E. M. Henley (World Scientific, Singapore, 1995), p. 67 and references therein.

[4] A. Kurylov, G. C. McLaughlin, and M. J. Ramsey-Musolf, Phys. Rev. D 63, 076007 (2001).

[5] M. J. Ramsey-Musolf, Phys. Rev. Lett. 83, 3997 (1999); 84, 5681(E) (2000).

[6] J. Engel, P. H. Frampton, and R. P. Springer, Phys. Rev. D 53, 5112 (1996).

[7] R. S. Conti and I. B. Khriplovich, Phys. Rev. Lett. 68, 3262 (1992).

[8] I. B. Khriplovich, Nucl. Phys. B352, 385 (1991).

[9] B. R. Davis, S. E. Koonin, and P. Vogel, Phys. Rev. C 22, 1233 (1980).

[10] SAMPLE Collaboration, S. P. Wells et al., Phys. Rev. C 63, 064001 (2001).

[11] N. F. Mott, Proc. R. Soc. London, Ser. A 135, 429 (1932).

[12] P. G. Blunden, W. Melnitchouk, and J. A. Tjon, Phys. Rev. Lett. 91, 142304 (2003).

[13] R. D. McKeown and M. J. Ramsey-Musolf, Mod. Phys. Lett. A 18, 75 (2003).

[14] P. A. M. Guichon and M. Vanderhaeghen, Prog. Part. Nucl. Phys. 41, 125 (1998).

[15] F. Maas, talk given at ECT*, Trento, Italy, April 2004, and F. Maas (private communication).

[16] SLAC E158 Collaboration, P. L. Anthony et al., hep-ex/
0312035; K. Kumar and Y. Kolomensky (private communication).

[17] A. O. Barut and C. Fronsdal, Phys. Rev. 120, 1871 (1960).

[18] L. L. DeRaad and Y. J. Ng, Phys. Rev. D 10, 683 (1974); 10, 3440 (1974); 11, 1586 (1975).

[19] L. Dixon and M. Schreiber, hep-ph/040222.

[20] G. G. Simon, C. Schmitt, F. Borkowski, and V. H. Walther, Nucl. Phys. A333, 381 (1980).

[21] R. K. Bhaduri, Models of the Nucleon (Addison-Wesley, Redwood City, CA, 1988).

[22] A. De Rujula, J. M. Kaplan, and E. De Rafael, Nucl. Phys. B35, 365 (1971).

[23] G.'t Hooft and M. Veltman, Nucl. Phys. B153, 365 (1979).

[24] G. Passarino and M. Veltman, Nucl. Phys. B160, 151 (1979).

[25] S. L. Zhu, S. Puglia, and M. J. Ramsey-Musolf, Phys. Rev. D 63, 034002 (2001).

[26] P. van Nieuwenhuizen, Nucl. Phys. B28, 429 (1971).

[27] A. Afanasev, I. Akushevich, and N. P. Merenkov, hep-ph/ 0208260.

[28] Y. C. Chen, A. Afanasev, S. J. Brodsky, C. E. Carlson, and M. Vanderhaeghen, hep-ph/0403058.

[29] B. Pasquini, D. Drechsel, M. Vanderhaeghen, M. Gorchtein, and A. Metz, in Dispersion Relations in Virtual Compton Scattering, edited by Y. I. Makdisi, A. U. Luccio, and W. W. Mackay, AIP Conf. Proc. No. 675 (AIP, New York, 2003), p. 646.

[30] J. Bernabeu and J. A. Penarrocha, Phys. Rev. D 22, 1082 (1980).

[31] D. Bardin, Lectures at the European School of High Energy Physics, Slovakia, 1999.

[32] B. Pasquini and M. Vanderhaeghen, hep-ph/0405303.

[33] A. V. Afanasev and N. P. Merenkov, hep-ph/0406127.

[34] A. Afanasev and N. P. Merenkov, hep-ph/0407167. 